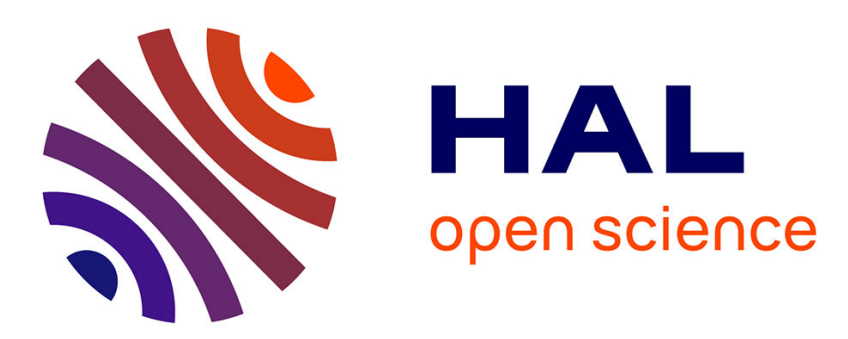

\title{
Sonoporation-enhanced chemotherapy significantly reduces primary tumour burden in an orthotopic pancreatic cancer xenograft
}

Spiros Kotopoulis, Anthony Delalande, Mihaela Popa, Veronika Mamaeva, Georg Dimcevski, Odd Helge Gilja, Michiel Postema, Bjørn Tore Gjertsen, Emmet Mc Cormack

\section{To cite this version:}

Spiros Kotopoulis, Anthony Delalande, Mihaela Popa, Veronika Mamaeva, Georg Dimcevski, et al.. Sonoporation-enhanced chemotherapy significantly reduces primary tumour burden in an orthotopic pancreatic cancer xenograft. Molecular Imaging and Biology, 2014, 16 (1), pp.53-62. 10.1007/s11307013-0672-5 . hal-03200844

\section{HAL Id: hal-03200844 https://hal.science/hal-03200844}

Submitted on 16 Apr 2021

HAL is a multi-disciplinary open access archive for the deposit and dissemination of scientific research documents, whether they are published or not. The documents may come from teaching and research institutions in France or abroad, or from public or private research centers.
L'archive ouverte pluridisciplinaire HAL, est destinée au dépôt et à la diffusion de documents scientifiques de niveau recherche, publiés ou non, émanant des établissements d'enseignement et de recherche français ou étrangers, des laboratoires publics ou privés. 
Title: Sonoporation enhanced chemotherapy significantly reduces primary tumour burden in an orthotopic pancreatic cancer xenograft.

Spiros Kotopoulis ${ }^{1,2}$, Anthony Delalande ${ }^{3, *}$, Mihaela Popa ${ }^{4, *}$, Veronika Mamaeva ${ }^{5}$ Georg 5 Dimcevski ${ }^{1}$, Odd Helge Gilja ${ }^{1,5}$, Michiel Postema ${ }^{2,1}$, Bjørn Tore Gjertsen ${ }^{5}$, Emmet McCormack ${ }^{5}$

${ }^{1}$ National Centre for Ultrasound in Gastroenterology, Haukeland University Hospital, Jonas Liesvei 65, 5021 Bergen, Norway

${ }^{2}$ Department of Physics and Technology, University of Bergen, Allégaten 55, 5007 Bergen, 10 Norway

${ }^{3}$ Centre de Biophysique Moléculaire, 45071 Orléans Cedex 2, France

${ }^{4}$ KinN Theapeutics AS, 5021 Bergen, Norway

${ }^{5}$ Department of Clinical Science, University of Bergen, Jonas Liesvei 65, 5021 Bergen, Norway

15 Shortened Title: Sonoporation enhanced chemotherapy of human pancreatic cancer.

Manuscript Category: Article

\section{Corresponding author details}

Emmet McCormack, $\mathrm{PhD}$

20 E-mail: emmet.mc.cormack@ med.uib.no

T: +4755975000

F: +4755972950

* These authors contributed equally 
Purpose: Adenocarcinoma of the pancreas remains one of the most lethal human cancers. The high mortality rates associated with this form of cancer are subsequent to late stage clinical presentation and diagnosis, when surgery is rarely possible and modest chemotherapeutic impact. Survival rates following diagnosis with advanced pancreatic cancer is very low; typical mortality 30 rates of $50 \%$ are expected within 3 months of diagnosis. However adjuvant chemotherapy improves the prognosis of patients even after palliative surgery, and successful newer neoadjuvant chemotherapeutical modalities have recently been reported. For patients whose tumours appear unresectable, chemotherapy remains the only option. During past two decades the nucleoside analogue gemcitabine has become the first-line chemotherapy for pancreatic

35 adenocarcinoma. In this study we aim to increase the delivery of gemcitabine to pancreatic tumours by exploring the effect of sonoporation for localised drug delivery of gemcitabine in an orthotopic xenograft mouse model of pancreatic cancer.

Experimental design: An orthotopic xenograft mouse model of luciferase expressing MIA PaCa-2 cells was developed exhibiting disease development similar to human pancreatic 40 adenocarcinoma. Subsequently, two groups of mice were treated with gemcitabine alone, gemcitabine combined with sonoporation, and saline treated mice were used as a control group. A custom made focused ultrasound transducer using clinically safe acoustic conditions in combination with SonoVue ${ }^{\circledR}$ ultrasound contrast agent was used to induce sonoporation in the localised region of the primary tumour only. Whole body disease development was measured using bioluminescence imaging and primary tumour development was measured using 3D ultrasound.

Results: Following just two treatments combining sonoporation and gemcitabine, primary tumour volumes were significantly lower than control groups. Additional therapy dramatically 
inhibited primary tumour growth throughout the course of the disease with median survival increases of up to $10 \%$ demonstrated in comparison to the control groups.

Conclusion: Combined sonoporation and gemcitabine therapy significantly impedes primary tumour development in an orthotopic xenograft model of human pancreatic cancer, suggesting additional clinical benefits for patients treated with gemcitabine in combination with sonoporation. 


\section{Introduction}

Pancreatic adenocarcinoma (PA) morbidity and mortality remains almost unchanged over the past two decades. Available statistical predictive data estimate 45,220 new cases and 38,460 deaths due to PA in 2013 [1]. This type of cancer can rarely be treated radically, and the overall survival rate is less than $4 \%$ [2]. When surgical intervention is possible survival rates can increase to median of 36 months and surpass 5 years [3]. However, adjuvant chemotherapy improves the prognosis of the patients even after palliative surgery, and successful newer neoadjuvant chemotherapeutical modalities have recently been reported [4]. Patients with unresectable tumours undergo chemotherapy and chemoradiation but should be spared the morbidities of surgical resection [5]. Most of the patients with advanced PA have about a year to live after diagnosis [6-7] and even increase in survival for a few months matters in the pancreatic cancer field. Gemcitabine, a nucleoside analogue, is considered the most effective chemotherapeutic treatment of pancreatic cancer in terms of both response rate and median overall survival [8-9]. The benefit it brings is relatively modest, as it does not improve the prognosis much, with a median overall survival of less than 6 months and has rather pronounced systemic side effects [10]. Gemcitabine is approved by the FDA as a single agent, as the first-line treatment for patients with locally advanced or metastatic adenocarcinoma of the pancreas.

Ultrasound is most widely known for its clinical use in diagnostic imaging and modern diagnostic ultrasonography employs contrast agents to improve the signal-to-noise ratio of blood [11-12]. These ultrasound contrast agents typically consist of perfluorocarbon-filled lipid-shelled microbubbles in the size range of $2-10 \mu \mathrm{m}$ (Table 1) [13] When excited with ultrasound, these microbubbles scatter the incoming ultrasound and volumetrically oscillate generating their own ultrasonic pulse. This results in various complex and controllable actions that can be taken 
advantage of for therapeutic purpose [14], e.g. sonoporation-enhanced therapy [15-20]. Sonoporation is the transient formation of nanometre-sized pores in cell membranes by the use of ultrasound or ultrasound and microbubbles allowing for intracellular uptake of drugs or genes $[20]$.

85

Previous research has elaborated six common mechanistic effects of sonoporation mediated drug and gene delivery [20-26] including push, pull, shear, jetting, inertial cavitation and translation. While "push", "pull", "shearing" and "translation" occur at all acoustic amplitudes resulting in perturbation of the cell membrane, "jetting" [27] and "inertial cavitation"

90 [28] result in partial destruction of the cell membrane which only occur at high acoustic amplitudes. High acoustic amplitudes employed in these studies surpass current ultrasound safety guidelines with the introduction of a cavitation nucleation agent, i.e., microbubbles [29-31]. Furthermore, a large majority of these studies only evaluate the effect of sonoporation in vitro [17, 32-34], in subcutaneous models [35] or for the efficacy of gene delivery [16, 32-33, 36].

95 Ultimately in a sonoporation therapeutic approach the goal must be to employ acoustically safe amplitudes but still impact translation of microbubbles and therapeutic across the cell membrane. Thus, "translation" sonoporation offers the preferred clinical modality resulting in microbubbles being forced inside a cell leaving a small pore that re-seals itself, occurring at low, clinically safe acoustic amplitudes.

100

Preclinical drug development in xenograft models of PA has historically been reliant upon subcutaneous inoculation of PA cells in the flanks of immunodeficient mice. However, subcutaneous models are not sufficiently representative of the clinical paradigm of PA, particularly with respect to metastasis development and drug response [37-38]. Using an 105 orthotopic model provides many advantages such as the attempts to target local invasion occur at 
a much more clinically relevant site and blood flow and vascularisation of the tumours closer mimic the human model. Taking into account the importance of an orthotopic model, in this study we aimed to investigate the potential of sonoporation for enhanced localised drug-delivery of a clinical chemotherapeutic (gemcitabine) to the primary tumour of PA in an orthotopic model.

Here we describe the development of an orthotopic PA mouse model exhibiting very similar characteristics to its human counterpart. A custom ultrasound transducer was used to induce sonoporation using clinically approved microbubbles (SonoVue ${ }^{\circledR}$ ) at an extremely localised area using identical acoustic settings shown to induce sonoporation in our previous

115 work [20, 25]. To follow the disease progression, tumour volume was measured weekly using 3D ultrasound and optical imaging was used to observe whole body disease development. Two treatments with combined gemcitabine and sonoporation resulted in statistically significant lower primary tumour volume. This statistical difference was persistent for the further duration of the study. Furthermore bioluminescence imaging indicated that there was a delay in the onset of metastasis in responding mice.

\section{Materials and Methods}

\section{Ultrasonic treatment conditions}

A custom-made single-element ultrasound transducer consisting of a 25-mm spherically focused element with a geometric focus of $44 \mathrm{~mm}$ (Precision Acoustics Ltd., Dorchester, UK) was used as the treatment probe. To ensure correct acoustic alignment with the pancreas depth and location a custom adaptor was designed and built based on the transducer dimensions and beam profile. The adaptor was filled with distilled water and an $80-\mu \mathrm{m}$ thick nitrile membrane 
was used at the contact surface to ensure maximum beam propagation. Figure 1a shows the design of the transducer and adaptor.

In order to drive the ultrasound transducer a $40 \%$ duty cycle sine wave was generated by an AFG3102, function generator (Tektronix, Inc., Beaverton, OR, USA) and amplified by a

135 2100L amplifier (Electronics \& Innovation Ltd., Rochester, NY, USA). The acoustic field was calibrated with the acoustic adaptor in place using an automated 3D scanning chamber with a 50$\mu \mathrm{m}$ resolution and a calibrated HGL-200 hydrophone (Onda, Sunnyvale, CA) in degassed water. Based on our previous in-vitro findings [20, 25] the acoustic settings shown in Table 2 were used to treat the pancreatic tumour.

140

\section{Ultrasound contrast agent / microbubbles}

A clinically employed ultrasound contrast agent $\left(\right.$ SonoVue ${ }^{\circledR}$, Bracco Imaging S.p.A, Milano, Italy) was prepared according the manufacturers instructions. This results in phospholipid shelled microbubbles containing an $\mathrm{SF}_{6}$ gas core with a mean diameter of $2.5 \mu \mathrm{m}$ at

145 concentrations between $1 \times 10^{8}-5 \times 10^{8}$ microbubbles per $\mathrm{mL}$ [39]. The microbubbles were prepared immediately prior to treatment of the first mouse and the vial was gently agitated prior to each treatment to ensure a homogenous concentration.

\section{Cell lines and cell culture}

The human pancreatic adenocarcinoma MIA PaCa-2 cell line were kindly provided by Dr. Anders Molven (The University of Bergen, Norway). The cells were retrovirally transfected using Phoenix cells (LGC Standards AB, Boras, Sweden) and a luciferase encoding plasmid. High luciferase expressing cells were selected by puromycin $(2.5 \mu \mathrm{g} / \mathrm{ml})$. Cells were cultured in DMEM (Sigma-Aldrich Co. LLC. St. Louis, MO) complemented by 10\% FBS, 2.5\% FHS, 100 $155 \mu \mathrm{g} / \mathrm{ml}$ penicillin-streptomycin and $2 \mathrm{mM}$ L-glutamine (Sigma-Aldrich). 


\section{Animals}

All experiments were approved by The Norwegian Animal Research Authority and conducted according to The European Convention for the Protection of Vertebrates Used for

160 Scientific Purposes. NOD-scid IL2r $\gamma^{\text {null }}$ (NSG) mice (Gades Institute, University of Bergen. Originally a generous gift of Prof. Leonard D. Shultz, Jackson Laboratories, Bar Harbour, Maine, USA). During surgery, depilation and imaging, mice were anaesthetized with $2 \%$ isofluorane (Isoba® vet, Intervet, Kenilworth, NJ).

\section{Orthotopic xenograft model}

Animals were fully anesthetized with $250 \mathrm{mg} / \mathrm{kg}$ tribromoethanol diluted in 2 methyl-2 butanol, $12.5 \mathrm{mg} / \mathrm{ml}$ (Sigma-Aldrich), and placed on a heating pad in dorsal recumbency. Hair was removed by shaving and the abdomen washed with iso-betadine and $70 \%$ alcohol. A small incision $(0.5 \mathrm{~cm})$ in the abdomen was made below the last rib on the left side, parallel to linea

170 alba. The pancreas was exteriorized and the cells $\left(1 \times 10^{6}\right.$ MIA PaCa- $\left.2^{\text {luc }}\right)$ suspended in $20-\mu \mathrm{L}$ phosphate buffered saline was injected using a 30-gauge needle. After placing the pancreas back in the original position, the muscles and the skin were sutured with Ethilon II 5-0 polyamide sutures (Ethicon, Inc., Somerville, NJ). Prior to return to their holding cages, animals were placed under heat lamps for approximately $30 \mathrm{~min}$, and monitored for any postoperative complications.

175 All animals were euthanized following institutional guidelines.

\section{Optical imaging}

The mice were anaesthetised and injected intraperitoneally (IP) with $150 \mathrm{mg} / \mathrm{kg}$ Dluciferin (Biosynth AG, Staad, Switzerland) 10 minutes prior to ventral and dorsal imaging using an In-Vivo FX molecular imaging system (Carestream Health, Inc., Rochester, NY). Total 
bioluminescence values were measured using manual ROIs in the Carestream MI software (Standard edition, v.5.0.6.20, Carestream Health, Inc.). Two types of ROIs were used, one whole abdomen and one locally in the primary tumour area.

\section{High-resolution ultrasonic imaging}

The mice were briefly re-anaesthetised and placed on the mouse handling table set at $40^{\circ} \mathrm{C}$ in dorsal recumbrency. The abdominal hair on the mice was removed using depilatory cream. A Vevo2100 ultrasound scanner combined with a MS250 (13-24 MHz) probe and 1D stage (VisualSonics Inc., Ontario, Canada) was used to capture 3D images of the primary tumour. The enhanced abdominal imaging mode in B-mode and 3D mode was used. Doppler imaging was also used to aid primary tumour identification. Respiration gating was used to capture the 3D images. The tumours were measured manually using parallel segmentation in the Vevo2100 software (v1.3.0, VisualSonics Inc.).

\section{Treatment protocol}

Three weeks following xenotransplantation, mice were randomly separated into 3 groups; control $(n=3)$, gemcitabine alone $(125 \mathrm{mg} / \mathrm{kg}$, Q7D, $n=3)$ and combined treatment group, employing SonoVue ${ }^{\circledR}(50 \mu \mathrm{L})$, and gemcitabine $(125 \mathrm{mg} / \mathrm{kg}, \mathrm{Q} 7 \mathrm{D}, n=4)$. Therapy was initiated on week 3 and continued weekly for a total of 8 cycles. Mice were euthanized when they started

200 to demonstrate progressive weight loss below $10 \%$ from initial body weight, jaundice and lethargy.

Control mice received $200-\mu \mathrm{L}$ saline (IP) post-imaging procedures. The gemcitabine group were treated with gemcitabine $(125 \mathrm{mg} / \mathrm{mg}$, i.p.) once weekly whilst combination treated animals received gemcitabine $\left(125 \mathrm{mg} / \mathrm{mg}\right.$, i.p.) and Sonovue ${ }^{\circledR}$ ultrasound contrast agent $(50-\mu \mathrm{L}$, i.v.) 30 minute later. Immediately after Sonovue ${ }^{\circledR}$ injection the mouse was placed on a heated 
table at $37^{\circ} \mathrm{C}$ and ultrasound was applied for a total of $10 \mathrm{~min}$ (4 min total cumulated ultrasound time). Figure $1 \mathrm{~b}$ shows how the mice and therapeutic transducer were positioned for the treatment.

\section{Histopathology}

Organ samples collected following euthanasia were placed in $4 \%$ paraformaldehyde for paraffin-embedding. Sections were stained with hematoxylin and eosin (H\&E) and results were analysed by standard light microscopy (Nikon Eclipse Ti, Nikon Instruments Europe BV, Amsterdam, Netherlands) by an experienced pathologist.

\section{Statistics}

Three different observers measured the primary tumour volume where two were blind to the groups. Results were expressed as the mean values \pm SEM. Comparisons between groups were made using a two-tailed unpaired Student t-test. Survival data was analysed using a Logrank (Mantel-Cox) test. Differences where $p<0.05$ were considered statistically significant. Statistics were analysed using GraphPad PRISM® v5.0a (GraphPad Software Inc., La Jolla, CA) software.

\section{Results}

\section{Transducer validation and characterisation}

The therapeutic transducer was fabricated in order to induce sonoporation in a very localised region generating a very precise and sharp focal (treatment) zone. The 3D field scan validated the beam profile indicating a focus of $\varnothing 4.0 \mathrm{~mm}$ and 22-mm long (Fig. 1c). A small 230 focus was chosen to ensure that only the primary pancreatic tumour was treated, as only this 
region would receive the ideal dose of ultrasound. The non-linearity ratio was 1.00 ensuring there were no shockwaves generated in the acoustic field prohibiting any other adverse effects seen by high-intensity ultrasound treatments and preventing microbubble destruction. Furthermore the adaptor reduced the side lobe intensity allowing for a Gaussian sound intensity profile [40].

235 By creating such a controllable sound field, with such a precise focus (treatment zone), we can clearly dictate the location and size of the treatment area, without treating the organs behind or in front of the pancreatic tumour.

\section{Pancreatic cancer xerograph model development}

240 Following inoculation of $1 \times 10^{6}$ MIA PaCa- $2^{\text {luc }}$ cells in the head of pancreas the mouse developed PA similar to that seen in the human counterpart. Three weeks after inoculation the primary tumour could be visualised by bioluminescence and high-resolution ultrasound (Fig. 2a and 4b respectively). Metastasis was evident 5-6 weeks after inoculation, as demonstrated by the increase of bioluminescence in the whole abdomen when compared to the primary tumour (Fig.

245 2b). After 8 weeks, metastases have disseminated throughout the whole abdomen and in particular the liver, and in 10 weeks it has engulfed the whole abdomen. The disease developed in a similar fashion to that seen in human patients with an exponential growth of the primary tumour with dense vascular encapsulation. Histology verified that this was a very aggressive form of PA characterised by the nuclear polymorphism, atypical mitosis and invasive growth pattern (Fig 2c).

$\underline{\text { Sonoporation of gemcitabine with microbubbles is safe in a preclinical model of pancreatic }}$ $\underline{\text { adenocarcinoma }}$

In order to evaluate the safety and efficacy of combined sonoporation and gemcitabine therapy, mice $(n=10)$ were inoculated and tumour and metastatic development monitored by 3D ultrasound and bioluminescent imaging. Primary tumour development was evident at 3 weeks by 
bioluminescence and 3D ultrasonic imaging, when mice were randomized into 3 groups. Mice weights were recorded every two days to monitor any adverse effects of the treatment. Following treatment a slight weight loss was recorded in all groups, with subsequent weight recovery following treatment. No significant differences in weight were seen between all three groups

260 (Supplementary Figure 1), and no additional ill effects were noted, suggesting sonoporation combined with chemotherapy was safe in this preclinical setting.

265 Combined Gemcitabine plus sonoporation significantly inhibits primary pancreatic adenocarcinoma tumour growth

While it was anticipated that therapy might have an impact upon the metastatic pattern of this very aggressive disease the ultimate goal remained to evaluate the effect of therapy within the sonoporation field. Thus, we evaluated the primary pancreatic tumours response to therapy 270 by tumoural bioluminescence imaging (Fig. 3) and 3D ultrasonic imaging (Fig. 4). Quantification of bioluminescence output from the primary tumour revealed reduced photonic flux in combination and gemcitabine treated mice than controls (Fig. 3a, $p=0.0003$ and 0.0019 respectively), however scattering of biophotonic light from adjacent metastasis inevitably contaminated the primary tumour photonic quantification. However, it was encouraging to see

275 lower bioluminescence at the primary site compared with controls.

In order to directly quantify primary tumour effects employing 3D ultrasound, we exploited Doppler imaging, which revealed dense vascularisation encapsulating the primary tumour (Fig. 4a-1 and 4a-2). Under high-frequency ultrasound, the primary tumour was seen as an anechoic 280 region (Fig. 4a-3) indicating a very homogenous cellular structure; this was also validated from 
post-study histology samples (Fig. 6). This was used to generate 3D volumes of primary tumours (Fig. 4a and b). Comparing tumour volumes from week 5 (Fig. 4c) onwards a statistically significant difference between the combined treatment group and the gemcitabine group versus control was seen. There was no statistical difference in primary tumour volume when comparing 285 the gemcitabine group to the control group, however statistically significant differences were observed between the combined treatment group versus both control $(p<0.05-0.001)$ and gemcitabine only groups $(p<0.05-0.001)$.

Combined treatment prolongs survival in an orthotopic xenograft model of pancreatic

$\underline{\text { adenocarcinoma }}$

Although whole-body bioluminescent imaging revealed a slower onset of metastatic development in combined treated animals (from weeks 6-8, Fig 3c) with lower bioluminescence observed at weeks 9 and 10, all animals eventually succumbed to metastatic disease. While disseminated metastasis of the liver and abdomen were noted in control and to a lesser extent 295 gemcitabine treated animals from weeks 7 to 10 , combination treated mice demonstrated restricted metastatic phenotype mostly restricted to the liver (Fig. 3c). Histopathology indicated a very aggressive form of PA with nuclear polymorphism, atypical mitosis and an invasive growth pattern. However, the combination treated group showed a less invasive growth pattern seen at the border between normal pancreatic and tumour tissue (Fig. 5). Furthermore, the combination and gemcitabine treated group demonstrated an increase in survival compared to the gemcitabine alone group with median survivals of 88 and 84 days respectively ( $p=0.0570$, Fig. 6) whilst the median survival of the control group was 80 days. 


\section{Discussion}

Sonoporation in combination with gemcitabine was employed to treat orthotopic PA xenografts resulting in a significant reduction of tumour growth, inhibited metastatic

310 development and increased survival when compared to controls. These results indicate potential to enhance localised drug delivery of chemotherapeutics, in consequence reducing the systemic toxic side effects and increasing local drug delivery. In this study we have used very low acoustic conditions that comply with current clinical safety regulations with the inclusion of a contrast agent [29-31] allowing for easy translation from pre-clinical to clinic studies. Clinical increase of

315 local neoadjuvant chemotherapeutic exposure by sonoporation should reduce the growth rate of the primary tumour and allow for the possibility of surgical resection and increase survival [3].

While gemcitabine therapy is the current standard of care in the therapy of pancreatic cancer, its clinical effect is modest, only extending survival by one month [41]. Similarly, in our preclinical study, gemcitabine improved survival over controls modestly; with both cohorts

320 exhibiting disseminated metastatic disease. Addition of sonoporation to gemcitabine therapy of the primary tumour not only further increased survival times, but also limited the dissemination of the disease. As such this may add a clinical benefit in limiting primary tumour growth making resection and curable treatment, possible.

Treatment could only be instigated once the tumour could be accurately located and

325 correlated to a physical location on the mouse, only possible 3 weeks following implantation. It was important to be able to unequivocally locate the tumour in 3-dimensions to accurately position the therapeutic probe. As a result, it is not inconceivable that within 3 weeks micrometastasis may have already seeded, undetectable with either imaging modality, prior to initiation of therapy. 
Further development of these pre-clinical studies should include near-infrared optical imaging agents [42-43] to improve primary tumour delineation. In addition, employing Multispectral Optoacoustic Tomography (MSOT) or Photoacoustic imaging (PA) would permit 3D tumour localisation at an earlier stage. Despite only using ten mice we required an extensive 335 skilled workforce to perform all the imaging in minimal time whilst still allowing the animals to recover. This resulted in a low power to statically analysis. However, the enabling technologies and imaging applications developed in this project should further serve development of this concept in treating patient derived xenografts surgical biopsies as they become available. Although individual mice will be treated at different times, similar to clinical trial, imaging of the 340 dense vasculature encapsulating the primary tumour (Fig. 4a) combined with optical imaging will aid standardisation of therapy initiation. Implanting and treating mice in this format will also reduce stress and enable greater numbers of animals to be treated.

The encapsulating vascularisation, though maintaining tumour nutrition seems beneficial

345 for the treatment with sonoporation as the microbubbles have better access to the tumour mass, and clinical contrast enhanced ultrasonography demonstrates that microbubbles are not adversely affected by high intratumoral pressures [44]. Developments that may further increase the efficacy of sonoporation would be to introduce a drug into these microbubbles creating anti-bubbles [45] and then inducing sonoporation. In this case the toxic chemotherapeutic would only be delivered 350 to the cancer cells. This would ensure maximum efficacy and reduce the side effects of the systemic chemotherapeutics.

As sonoporation has been shown to improve the drug delivery to localized areas, other drug treatments in combination with sonoporation should be explored. Several new drugs are being investigated in-vitro and in pre-clinical trials that have indicated high effectiveness in 
treating pancreatic cancer cells [46-48]; evaluating the efficiency these drugs in combination with the benefits of sonoporation needs to be further studied.

Following these results we have commenced a Phase I study to evaluate the toxicity and/or efficacy of gemcitabine combined with inducing sonoporation in the clinic on pancreatic cancer tumours (2011/1601/REK).

\section{Conclusion}

In this study we have developed a custom ultrasound transducer to induce sonoporation in a very localised region required for preclinical studies and a bioluminescent model of pancreatic

365 adenocarcinoma. We demonstrated that by inducing sonoporation at the location of an orthotopic pancreatic xenograft primary tumour in combination with the chemotherapeutic gemcitabine we are able to significantly inhibit the tumour growth compared with control or gemcitabine treated mice, even in cohorts with low sample number. Our results indicate that it is possible to enhance the efficacy of current chemotherapeutics with the simple addition of low-cost ultrasound contrast agents and a sound source. To further validate the clinical relevance of this study, repeat studies of primary patient derived xenografts, possibly with the addition of near-infrared contrast reagents and MSOT imaging should be considered. On the basis of these preclinical results the combination of gemcitabine and sonoporation is currently undergoing Phase I clinical trial at our centre.

\section{Acknowledgements}

This work was supported by the Norwegian Cancer Society (grant number 421828, 732200); the Western Health Board of Norway (grant numbers 911182, 911789) and the Bergen Research Foundation. The study was supported by MedViz (http://medviz.uib.no/), an interdisciplinary 
research cluster from Haukeland University Hospital, University of Bergen and Christian

Michelsen Research AS. We would like to thank Lars Helgeland (Section for Pathology, The Gade Institute, University of Bergen) for his advice on the histology. 


\section{Tables}

385 Table 1: List of the most common ultrasound contrast agents [49]. Bold names denote contrast agents clinically approved for human use.

\begin{tabular}{|c|c|c|}
\hline Contrast agent & Mean diameter $(\mu \mathrm{m})$ & Gas \\
\hline AI-700 & 2 & $\mathrm{C}_{4} \mathrm{~F}_{10}$ \\
\hline BR14 & 3.0 & $\mathrm{C}_{4} \mathrm{~F}_{10}$ \\
\hline CARDIOsphere ${ }^{\circledR}$ & 4.0 & $\mathrm{~N}_{2}$ \\
\hline Definity® & $1.1-3.3$ & $\mathrm{C}_{3} \mathrm{~F}_{8}$ \\
\hline EchoGen ${ }^{\circledR}$ & $2-5$ & $\mathrm{C}_{5} \mathrm{~F}_{12}$ \\
\hline Imagent ${ }^{\circledR}$ & 6.0 & $\mathrm{C}_{6} \mathrm{~F}_{14} / \mathrm{N}_{2}$ \\
\hline MicroMarker $^{\mathrm{TM}}$ & 2.5 & $\mathrm{C}_{4} \mathrm{~F}_{10} / \mathrm{N}_{2}$ \\
\hline Optison $^{\mathrm{TM}}$ & $2.0-4.5$ & $\mathrm{C}_{3} \mathrm{~F}_{8}$ \\
\hline Quantison $^{\mathrm{TM}}$ & 3.2 & Air \\
\hline SonoVue® & 2.5 & $\mathrm{SF}_{6}$ \\
\hline Sonazoid & $2.4-3.6$ & $\mathrm{C}_{4} \mathrm{~F}_{10}$ \\
\hline
\end{tabular}


Table 2: Acoustic settings used to enhance drug delivery.

\begin{tabular}{|c|c|c|c|c|}
\hline $\begin{array}{l}\text { Centre } \\
\text { frequency } \\
(\mathrm{MHz})\end{array}$ & $\begin{array}{l}\text { Duty cycle } \\
(\%)\end{array}$ & $\begin{array}{l}\text { Mechanical } \\
\text { Index }\end{array}$ & $\begin{array}{l}\text { Acoustic } \\
\text { power ISPTA } \\
\left(\mathbf{m W} / \mathbf{c m}^{2}\right)\end{array}$ & $\begin{array}{l}\text { Peak-negative } \\
\text { acoustic } \\
\text { pressure (MPa) }\end{array}$ \\
\hline 1.00 & $\begin{array}{l}40 \text { (40 cycles } \\
\text { every } 100 \mu \mathrm{s})\end{array}$ & 0.2 & 688 & 0.200 \\
\hline
\end{tabular}

390 
Figures

395

Figure 1: Treatment setup using custom made ultrasound transducer.

Focal region aligned with pancreatic tumour (Panel b); Verification of the acoustic field using a 3D scanning tank (Panel c). The $-3 \mathrm{~dB}$ boundary defining the beam size is indicated by the yellow lines (Normalised intensity of 0.5 ).

400 
Figure 2: Disease development of NOD/SCID IL2r $\gamma^{\text {null }}$ mice inoculated with MIA PaCa- $2^{\text {Luc }}$ 405 cells $\left(1 \times 10^{6}\right.$ cells Day 0$)$ and treated with gemcitabine; gemcitabine, microbubbles and ultrasound or control once a week. Panel a shows bioluminescence images from week 2 to week 10. Panel $b$ shows the net photon count of the whole abdomen and primary tumour on a logarithmic scale. Panel c shows the histology images after the mice were sacrificed. 
Figure 3: Primary tumour (Panel a) and whole abdomen (Panel b) bioluminescence count of MIA PaCa-2 ${ }^{\text {Luc }}$ model treated with gemcitabine, gemcitabine microbubbles and ultrasound or control once a week. Optical bioluminescence imaging of representative mouse of each group

415 (Panel c). No statistical difference between the gemcitabine and sonoporation treated group can be seen. The control group had a much higher total bioluminescence count at the final stages of the disease progression. 
Figure 4: Primary pancreatic tumour volume measured by $3 \mathrm{D}$ ultrasound of MIA PaCa-2 $2^{\text {luc }}$ model treated with gemcitabine; gemcitabine microbubbles and ultrasound or control once a week. Panel A shows how the primary tumour was detected and 3D volume was measured. Using colour Doppler the feeding arteries of the tumour and kidney can be easily distinguished in both $4302 \mathrm{D}$ and 3D (Panel a1 \& a2 respectively). The primary tumour can then be manually contoured throughout the 3D stack (Panel a3). The primary tumour can then be visualised within the 3D ultrasound image (Panel a4) or on its own (Panel a5). 3D tumour volume over time can be visualised and compared. The $3 \mathrm{D}$ volumes are visualised in Panel $\mathrm{b}$ and mean volume $\pm \mathrm{SEM}$ are shown in Panel c. After two treatments a statistically significant difference can be seen between 435 the combined treatment group and the gemcitabine alone and/or control group. 
Figure 5: Survival data of MIA $\mathrm{PaCa}-2^{\text {luc }}$ model treated with gemcitabine; gemcitabine microbubbles and ultrasound or control. A slight increase in survival time can be seen between the groups but was not statistically significant.

445 
Figure 6: Histology images showing the invasive border between normal and tumour tissue in the control group and the less invasive border in the seen in the sonoporation treated group indicated by the asterisks $(*)$. As the samples were taking at different time point a very 450 aggressive form of pancreatic cancer was observed in all cases. 


\section{Conflict of Interest}

The authors have no conflict of interest. 


\section{References}

460 1. American Cancer Society Cancer Facts and Figures 2013. http://www.cancer.org/acs/groups/content/@epidemiologysurveilance/documents/document/acsp c-036845.pdf.

2. Greenlee RT, Murray T, Bolden S, Wingo PA (2000) Cancer statistics, 2000. CA Cancer J Clin 50:7-33.

465 3. Chen EL, Prinz RA (2007) Long-term survival after pancreatic cancer treatment. Am J Surg 194:S127-S130.

4. Hartlapp I, Muller J, Kenn W, et al. (2013) Complete Pathological Remission of Locally Advanced, Unresectable Pancreatic Cancer (LAPC) after Intensified Neoadjuvant Chemotherapy. Onkologie 36:123-125.

470 5. Tempero MA, Arnoletti JP, Behrman SW, et al. (2012) Pancreatic Adenocarcinoma, version 2.2012: featured updates to the NCCN Guidelines. J Natl Compr Canc Netw 10:703-713. 6. Neoptolemos JP, Stocken DD, Friess H, et al. (2004) A randomized trial of chemoradiotherapy and chemotherapy after resection of pancreatic cancer. $\mathrm{N}$ Engl $\mathrm{J}$ Med 350:1200-1210.

475 7. Neoptolemos JP, Dunn JA, Stocken DD, et al. (2001) Adjuvant chemoradiotherapy and chemotherapy in resectable pancreatic cancer: a randomised controlled trial. Lancet 358:15761585.

8. el-Kamar FG, Grossbard ML, Kozuch PS (2003) Metastatic pancreatic cancer: emerging strategies in chemotherapy and palliative care. Oncologist 8:18-34.

480 9. Marechal R, Bachet JB, Mackey JR, et al. (2012) Levels of gemcitabine transport and metabolism proteins predict survival times of patients treated with gemcitabine for pancreatic adenocarcinoma. Gastroenterology 143:664-674 e661-666. 
10. Camp-Sorell D (2010) Chemotherapy Toxicities and Management. In Cancer Nursing: Principles and Practice, Eds. Yarbro CH, Wujcik D, Gobel BH. Sudbury: Jones \& Bartlett.

485 11. Erchinger FG, Dimcevski G, Engjom T, Gilja OH (2011) Transabdominal ultrasonography of the pancreas: basic and new aspects. Imaging Med 3:412-422.

12. Simpson DH, Burns PN, Averkiou MA (2001) Techniques for perfusion imaging with microbubble contrast agents. IEEE Trans Ultrason Ferroelectr Freq Control 48:1483-1494.

13. Stride E, Saffari N (2003) Microbubble ultrasound contrast agents: a review. Proc Inst 490 Mech Eng H J Eng Med 217:429-447.

14. Postema MAB (2004) Medical Bubbles. Bergschenhoek: Michiel Postema.

15. Qiu Y, Zhang C, Tu J, Zhang D (2012) Microbubble-induced sonoporation involved in ultrasound-mediated DNA transfection in vitro at low acoustic pressures. J Biomech 45:13391345.

495 16. Suzuki R, Oda Y, Utoguchi N, Maruyama K (2011) Progress in the development of ultrasound-mediated gene delivery systems utilizing nano- and microbubbles. Journal of Controlled Release 149:36-41.

17. Nejad SM, R. Hosseini SH, Akiyama H, Tachibana K (2011) Optical observations of cell sonoporation with low intensity ultrasound. Biochem Biophys Res Co 413:218-223.

500 18. Kooiman K, Harteveld M, van der Steen AFW, de Jong N (2011) Sonoporation of endothelial cells by vibrating targeted microbubbles. J Control Release 154:35-41.

19. Wu J, Pepe J, Rincon M (2006) Sonoporation, anti-cancer drug and antibody delivery using ultrasound. Ultrasonics 44:E21-E25.

20. Delalande A, Kotopoulis S, Rovers T, Pichon C, Postema M (2011) Sonoporation at a low 505 mechanical index. Bub Sci Eng Tech 3:3-11.

21. van Wamel A, Bouakaz A, Bernard B, ten Cate F, de Jong N (2005) Controlled drug delivery with ultrasound and gas microbubbles. J Control Release 101:389-391. 
22. Emmer M, Borsboom J, Van Wamel A, Versluis M, de Jong N (2005) Optical investigation of ultrasound induced encapsulated microbubble oscillations: threshold and 510 hysteresis effects. Proc IEEE Ultrason Symp:981-984.

23. van Wamel A, Kooiman K, Harteveld M, et al. (2006) Vibrating microbubbles poking individual cells: drug transfer into cells via sonoporation. J Control Release 112:149-155.

24. Postema M, Kotopoulis S, Delalande A, Gilja OH (2012) Sonoporation: Why microbubbles create pores. Ultraschall in Med 33:97-98.

515 25. Delalande A, Bouakaz A, Renault G, et al. (2011) Ultrasound and microbubble-assisted gene delivery in Achilles tendons: long lasting gene expression and restoration of fibromodulin KO phenotype. J Control Release 156:223-230.

26. Delalande A, Kotopoulis S, Postema M, Midoux P, Pichon C (2013) Sonoporation: Mechanistic insights and ongoing challenges for gene transfer. Gene.

520 27. Gerold B, Rachmilevitch I, Prentice P (2013) Bifurcation of ensemble oscillations and acoustic emissions from early stage cavitation clouds in focused ultrasound. New Journal of Physics 15:033044.

28. Gerold B, Kotopoulis S, C. M, McGloin D, Postema M, Prentice P (2011) Lasernucleated acoustic cavitation in focused ultrasound. Rev Sci Instrum 82:044908.

525 29. Piscaglia F, Nolsoe C, Dietrich CF, et al. (2012) The EFSUMB Guidelines and Recommendations on the Clinical Practice of Contrast Enhanced Ultrasound (CEUS): update 2011 on non-hepatic applications. Ultraschall in Med 33:33-59.

30. $\mathrm{Ng} \mathrm{KH} \mathrm{(2002)} \mathrm{International} \mathrm{guidelines} \mathrm{and} \mathrm{regulations} \mathrm{for} \mathrm{the} \mathrm{safe} \mathrm{use} \mathrm{of} \mathrm{diagnostic}$ ultrasound in medicine. J Med Ultrasound 10:5-9.

530 31. British Medical Ultrasound Society (2000) Guidelines for the safe use of diagnostic ultrasound equipment. 
32. Fan Z, Kumon RE, Park J, Deng CX (2010) Intracellular delivery and calcium transients generated in sonoporation facilitated by microbubbles. Journal of Controlled Release 142:31-39.

33. Tlaxca JL, Anderson CR, Klibanov AL, et al. (2010) Analysis of in vitro Transfection by

535 Sonoporation Using Cationic and Neutral Microbubbles. Ultrasound Med Biol 36:1907-1918.

34. Qiu Y, Luo Y, Zhang Y, et al. (2010) The correlation between acoustic cavitation and sonoporation involved in ultrasound-mediated DNA transfection with polyethylenimine (PEI) in vitro. Journal of Controlled Release 145:40-48.

35. Czarnota GJ, Karshafian R, Burns PN, et al. (2012) Tumor radiation response

540 enhancement by acoustical stimulation of the vasculature. Proc Natl Acad Sci U S A 109:E20332041.

36. Lukianova-Hleb EY, Samaniego AP, Wen J, Metelitsa LS, Chang CC, Lapotko DO (2011) Selective gene transfection of individual cells in vitro with plasmonic nanobubbles. J Control Release 152:286-293.

545 37. Hoffman RM (1999) Orthotopic metastatic mouse models for anticancer drug discovery and evaluation: A bridge to the clinic. Invest New Drug 17:343-359.

38. Bibby MC (2004) Orthotopic models of cancer for preclinical drug evaluation: advantages and disadvantages. Eur J Cancer 40:852-857.

39. Greis C (2004) Technology overview: SonoVue (Bracco, Milan). Eur Radiol 14:P11-P15.

550 40. Lu JY, Zou H, Greenleaf JF (1994) Biomedical ultrasound beam forming. Ultrasound Med Biol 20:403-428.

41. Burris HA, 3rd, Moore MJ, Andersen J, et al. (1997) Improvements in survival and clinical benefit with gemcitabine as first-line therapy for patients with advanced pancreas cancer: a randomized trial. J Clin Oncol 15:2403-2413.

555 42. McCormack E, Mujic M, Osdal T, Bruserud O, Gjertsen BT (2013) Multiplexed mAbs: a new strategy in preclinical time-domain imaging of acute myeloid leukemia. Blood 121:e34-42. 
43. McCormack E, Silden E, West RM, et al. (2013) Nitroreductase, a near-infrared reporter platform for in vivo time-domain optical imaging of metastatic cancer. Cancer Res 73:1276-1286. 44. Ozawa Y, Numata K, Tanaka K, et al. (2002) Contrast-enhanced sonography of small 560 pancreatic mass lesions. J Ultrasound Med 21:983-991.

45. Poortinga AT (2012) Micron-sized antibubbles with tunable stability. Colloids Surf A: Physiochem Eng Aspects.

46. Zhang GN, Liang Y, Zhou LJ, et al. (2011) Combination of salinomycin and gemcitabine eliminates pancreatic cancer cells. Cancer Lett 313:137-144.

565 47. Michaelis M, Doerr HW, Cinatl J (2007) Valproic acid as anti-cancer drug. Curr Pharm Design 13:3378-3393.

48. Kunnumakkara AB, Guha S, Krishnan S, Diagaradjane P, Gelovani J, Aggarwal BB (2007) Curcumin potentiates antitumor activity of gemcitabine in an orthotopic model of pancreatic cancer through suppression of proliferation, angiogenesis, and inhibition of nuclear

570 factor-kappaB-regulated gene products. Cancer Res 67:3853-3861.

49. Postema M (2011) Fundamentals of medical ultrasonics. Milton Park, Abingdon, Oxon ; New York: Spon Press. 